\title{
Estrutura da comunidade de formigas (Hymenoptera: Formicidae) em quatro ambientes com diferentes níveis de perturbação antrópica
}

\author{
Junir A. Lutinski ${ }^{1, \bowtie}$; Cladis J. Lutinski²; Benedito Cortês Lopes ${ }^{3} \&$ Ana B. Barros De \\ MORAIS $^{1}$ \\ 1. Programa de Pós-Graduação em Biodiversidade Animal, Universidade Federal de Santa Maria, RS, Brasil. 2. Universidade \\ Federal da Fronteira Sul, Lab. de Biologia. Santa Catarina, Brasil. 3. Departamento de Ecologia e Zoologia, Centro de Ciências \\ Biológicas, Universidade Federal de Santa Catarina, Santa Catarina, Brasil.
}

\begin{abstract}
REsumo. A diversidade e a estrutura das comunidades biológicas são afetadas pela intensificação das atividades antrópicas. Este estudo avaliou a estrutura das assembleias de formigas de quatro tipos de ambientes ao longo de um gradiente de intensidade de perturbação antrópica em dez cidades situadas na região austral do Domínio Mata Atlântica. Os ambientes avaliados foram: fragmentos florestais, áreas verdes, escolas e centros de reciclagem. Foram realizadas duas amostragens durante o ano de 2011, uma em fevereiro e março e outra em outubro e novembro. Para a comparação das assembleias de formigas foi desenvolvida uma análise de rarefação baseada no número de ocorrências nos quatro tipos de ambientes. A composição das assembleias foi verificada através de um teste de aninhamento utilizando a métrica a NODF. A associação das espécies de formigas com os ambientes amostrados foi obtida por meio de uma Análise de Espécies Indicadoras (ISA). Foram encontradas diferenças significativas de riqueza e de abundância entre os ambientes, mostrando uma tendência de redução nos ambientes escolares e centros de reciclagem em relação aos fragmentos florestais e áreas verdes. Observou-se um padrão de aninhamento ao longo do gradiente, ou seja, as assembleias pertencentes aos ambientes sob maior pressão antrópica são subconjuntos da mirmecofauna dos ambientes mais conservados. Não houve alteração da composição das assembleias, e sim uma perda importante de espécies de um ambiente para outro. Os fragmentos florestais apresentaram o maior número de espécies indicadoras (12), seguido pelas áreas verdes (4) e escolas (3). Os resultados podem ajudar na implementação de políticas de desenvolvimento urbano e na elaboração de planos de conservação.
\end{abstract}

[Palavras-chave: áreas verdes urbanas, composição, conservação, fragmentos florestais, riqueza]

\begin{abstract}
Ant community structure (Hymenoptera: Formicidae) in four environments with different levels of human disturbance: The diversity and biological communities structure are affected by the intensification of human activities. This study evaluated the ant community structure from four types of environments with different levels of human disturbance intensity at ten cities in the southern region of the Atlantic Forest Biome. The environments were: forest fragments, green areas, schools, and recycling centers. Two samplings were conducted in 2011, one in February and March and another in October and November. To compare the ant assemblages, it was performed a rarefaction analysis based on occurrences number. The composition of the assemblages was evaluated by a nestedness test using the NODF metric. The association of ant species with the environments was obtained by an Indicator Species Analysis (ISA). Significant differences in richness and abundance were found between the environments, showing a downward trend in schools and recycling centers in relation to forest fragments and green areas. There was a nesting pattern along the gradient, ie the assemblies belonging to the high human pressure environments are subsets of the conserved ones. There was no change in the composition of the assemblies, but a significant loss of species from one environment to another. The forest fragments had the highest number of indicator species (12), followed by green areas (4) and schools (3). The results can help in the implementation of urban development policies and development of conservation plans.
\end{abstract}

[Keywords: composition, conservation, forest fragments, richness, urban green areas]

\section{INTRODUÇãO}

A diversidade e a estrutura das comunidades biológicas são afetadas pela intensificação das atividades antrópicas, especialmente devido à superexploração dos recursos naturais (Diamond 2012). O processo de urbanização é uma das principais ameaças à biodiversidade e causa da fragmentação de ecossistemas

Editor asociado: Alejandro G. Farji-Brener

$\bowtie$ junir@unochapeco.edu.br
(McKinney 2002; Toennisson et al. 2011), o que resulta em impacto sobre a riqueza e a abundância das espécies (Sanford et al. 2008). Este processo afeta a qualidade dos habitats remanescentes dentro do perímetro urbano ou próximo a ele, dificultando ou impedindo a dispersão de animais e plantas (Connor et al. 2002) e aumentando, diminuindo ou extinguindo populações locais (Morini et 
al. 2007; Dáttilo et al. 2011). A urbanização cria ainda ambientes relativamente homogêneos e força espécies nativas a se adaptarem a um ambiente muito diferente de um ecossistema natural. Esse processo faz com que muitas espécies especialistas se tornem localmente extintas e possibilita que poucas espécies generalistas se tornem abundantes e dominantes nesses ambientes, tolerando e se beneficiando das atividades humanas (Buczkowski \& Richmond 2012). Nesse contexto, é necessário conhecer a biodiversidade existente em ambientes sob diferentes graus de pressão antrópica e identificar padrões que ajudem na identificação de ambientes de interesse para a conservação.

Formicidae constitui um dos táxons de insetos mais diversificados (Hölldobler \& Wilson 1990), caracterizado pela ampla distribuição, riqueza e abundância nos ecossistemas terrestres (Alonso \& Agosti 2000). Representa um grupo ideal para estudos do efeito da urbanização sobre a estrutura de suas comunidades (López-Moreno et al. 2003). A fauna de formigas que habita áreas urbanas do bioma Mata Atlântica tem sido extensamente estudada nos últimos anos (Morini et al. 2007; Iop et al. 2009), entretanto, a mirmecofauna de muitas regiões ainda permanece totalmente desconhecida (Ulysséa et al. 2011). A riqueza e a abundância de formigas de ambientes residenciais e comerciais (Oliveira \& Campos-Farinha 2005; Iop et al. 2009), fragmentos florestais e áreas verdes (Pacheco \& Vasconcelos 2007) já foram alvos de estudos. Contudo, há uma carência de informações acerca das alterações na comunidade de formigas ao logo de gradientes de perturbação antrópica envolvendo ambientes situados em áreas urbanas.

Ambientes escolares localizados em áreas urbanas possuem uma quantidade limitada de cobertura vegetal. A vegetação existente é composta essencialmente por plantas ornamentais, exóticas e de pequeno porte usadas na formação de jardins enquanto que árvores raramente estão presentes. Este ambiente se caracteriza ainda pela presença de edificações, calçadas e estacionamentos e pela intensa atividade humana local. Os centros de reciclagem representam um extremo da ação antrópica em um ambiente urbano. São locais desprovidos de vegetação, recebem e armazenam grandes quantidades de lixo, suportam a drenagem de compostos diretamente para o solo e a queima constante de resíduos indesejáveis. Ainda contam com uma movimentação constante de pessoas e veículos (Lutinski \& Souza 2009). O conhecimento da estrutura das assembleias de formigas desses ambientes pode ajudar na elucidação dos fatores que favoreçam seu estabelecimento em cada um destes ambientes e ainda indicar espécies capazes de suportar condições extremas de impacto antrópico. Enquanto a maioria dos estudos se volta para o impacto negativo do processo de urbanização sobre as assembleias de formigas, uma nova tendência começa a mostrar que algumas espécies podem se beneficiar desse processo se tornando dominantes e assim podem predizer informações importantes sobre o ambiente em que se encontram (Pacheco \& Vasconcelos 2007; Stringer et al. 2009).

Este estudo buscou avaliar 1) a variação da riqueza e da abundância de formigas entre ambientes ao longo de um gradiente de perturbação antrópica, 2) se as espécies mais abundantes e dominantes em ambientes menos perturbados também assumem tal condição nos ambientes mais perturbados, 3) se a composição das assembleias de formigas varia em função dos ambientes amostrados, e 4) se há espécies de formigas com potencial de indicação para os ambientes avaliados.

\section{Material e Métodos}

Foram avaliadas as assembleias de formigas pertencentes a quatro tipos de ambientes classificados em um gradiente de intensidade de perturbação antrópica: elegemos fragmentos florestais como o tipo de ambiente sob menor perturbação, seguido por áreas verdes, escolas e centros de reciclagem no outro extremo do gradiente como o aquele sob maior perturbação. Foram amostrados 30 fragmentos florestais, 30 áreas verdes, 30 escolas e 19 centros de reciclagem distribuídos em dez cidades do estado de Santa Catarina, conforme Informação Suplementar, Tabela S1. As amostragens nos centros de reciclagem ficaram restritas à existência desse tipo de estabelecimento nas cidades. A distância mínima verificada entre uma réplica e outra foi de $300 \mathrm{~m}$. Esta distância mínima também foi respeitada entre réplicas de ambientes diferentes. As cidades encontram-se situadas em uma extensão de aproximadamente $300 \mathrm{~km}$ no sentido leste/oeste e de $80 \mathrm{~km}$ no sentido norte/sul.

Os fragmentos florestais estudados são remanescentes de Mata Atlântica, Floresta Ombrófila Mista e Floresta Estacional Semidecídua, possuem área média de 4.6 ha cada um e encontram-se situados no interior ou na periferia de suas respectivas cidades. Apresentam vestígios de atividades humanas, porém de baixo impacto 
como a existência de trilhas de passagem, a retirada de árvores mortas ou parte de árvores para uso como fonte de energia e a deposição eventual de lixo. Como áreas verdes foram consideradas praças e parques urbanos com área média de 2.06 ha. Arborizados predominantemente com espécies nativas da região, são locais utilizados para a prática de atividades físicas e de recreação. Verifica-se a existência de sub-bosque esparso constituído essencialmente por gramíneas e vegetação arbustiva. Os ambientes escolares possuem área média (incluindo áreas de edificações, jardins e calçadas) de 0.5 ha. A vegetação existente é composta predominantemente por gramíneas e plantas ornamentais, exóticas e de pequeno porte. Como centros de reciclagem foram avaliados espaços destinados ao recebimento, separação e armazenamento de materiais recicláveis (metais, plásticos, papel, vidro e outros). Esses ambientes possuem área média de 0.2 ha e se caracterizam pela ausência de vegetação e pelo acúmulo de materiais de diferentes origens (Lutinski \& Souza 2009).

\section{Amostragem}

Foram realizadas duas campanhas de amostragem durante o ano de 2011, uma em fevereiro e março e a outra em outubro e novembro. A metodologia consistiu no uso de iscas de sardinha e de glicose (10 iscas de $1 \mathrm{~g}$ de sardinha, 10 iscas de $1 \mathrm{ml}$ de glicose) e coleta manual por uma hora, em cada réplica e em cada campanha de amostragem. As iscas foram preparadas sobre retângulos de papel poroso e distribuídas de forma alternada sobre o solo equidistantes 10 metros entre si (Bestelmeyer et al. 2000; Lutinski et al. 2013a). As amostragens manuais foram conduzidas obedecendo a um percurso aleatório (Sarmiento 2003). Foi utilizado um total de 4360 iscas e empregado um total de $218 \mathrm{~h}$ de coleta manual. As campanhas foram conduzidas durante o dia (entre 08:00 e 17:00 h) com alternância dos turnos matutino e vespertino em cada réplica, por exemplo, se um dado fragmento foi amostrado na primeira campanha no período da manhã, na segunda campanha este foi amostrado no período da tarde.

Os espécimes coletados foram transportados ao Laboratório de Entomologia da Universidade Comunitária da Região de Chapecó (UNOCHAPECÓ), Chapecó, SC, Brasil, para a triagem. A identificação foi conduzida primeiramente no laboratório de Biologia de Formigas da Universidade Federal de Santa Catarina, Florianópolis, SC, Brasil, utilizandose chaves disponíveis. Posteriormente, foi feita comparação dos exemplares coletados com os espécimes tombados na coleção do Museu de Zoologia da Universidade de São Paulo (USP), São Paulo, SP, Brasil. O material testemunho foi depositado na coleção entomológica do Laboratório de Entomologia da UNOCHAPECÓ, Chapecó, SC, Brasil. Maior detalhamento das técnicas de identificação utilizadas neste estudo podem ser verificados em Lutinski et al. (2013b).

\section{Análise estatística}

A riqueza de formigas das réplicas de cada tipo de ambiente foi comparada através de um diagrama do tipo "boxplot" (Tukey 1977) construído no programa estatístico PAST (Hammer et al. 2001). Esta análise é exploratória e resume os dados em cinco números: valor mínimo, primeiro quartil, mediana, terceiro quartil e valor máximo. Possibilita a comparação de séries de dados pertencentes a diferentes categorias. As assembleias de formigas de cada tipo de ambiente foram comparadas também por meio de análises de rarefação baseadas no número de ocorrências (Gotelli \& Colwell 2001). Essa análise foi obtida utilizando-se o programa EcoSim 7 (Gotelli \& Entsminger 2001) e permite comparações de riqueza entre assembleias que diferem quanto à ocorrência de espécies (Melo et al. 2003).

A avaliação das espécies abundantes e dominantes foi realizada a partir de uma tabela comparativa onde foram listadas as cinco espécies mais frequentes nas amostras de cada tipo de ambiente. A frequência nas amostras foi definida como o número de vezes que uma dada espécie foi registrada em uma das iscas ou na coleta manual, desconsiderando o número de indivíduos amostrados. As ocorrências foram agrupadas nos quatro tipos de ambientes (categorias do gradiente) independentemente da réplica ou da cidade. Como "abundantes" foram consideradas as espécies que apresentaram as maiores frequências absolutas de ocorrências e como "dominantes" as que apresentaram frequência relativa maior que $10 \%$ (fr $>0.1$ ) (Novotný \& Basset 2000).

Para avaliar possíveis variações na composição das assembleias de formigas correspondentes a cada tipo de ambiente, estas foram submetidas a um teste de aninhamento utilizando a métrica NODF (métrica de aninhamento baseada em sobreposição e redução de riqueza) (Almeida-Neto et al. 2008) com o uso do programa estatístico $R$ (R Development Core Team 2011). Este teste teve como objetivo avaliar se a composição das espécies varia de um ambiente para outro, se há perda ou substituição de espécies. Um resultado significativo de aninhamento permite inferir que ambientes com menor riqueza podem ser classificados como subconjuntos de um ambiente com maior riqueza (Ulrich et al. 2009). Para a visualização do grau de aninhamento da comunidade, foi construída uma matriz de distribuição das espécies de acordo com Atmar \& Patterson (1993). Nesta matriz os ambientes são dispostos nas linhas, em ordem decrescente de acordo com a sua riqueza e as espécies são dispostas nas colunas de acordo com o número de ambientes em que ocorreram.

A associação das espécies de formigas com os ambientes amostrados foi obtida por meio de uma Análise de Espécies Indicadoras (ISA) (Dufrêne \& Legendre 1997), obtendo-se o valor de indicação para cada espécie. As espécies foram consideradas indicadoras quando obtiveram o valor de indicação $\geq 25 \%$ e probabilidade estatística $P=0.05$. Para 
esta análise foi utilizado o programa PC-Ord 5.31 (McCune \& Mefford 2006).

\section{Resultados}

Foi identificada uma riqueza total de 140 espécies de formigas nos quatro ambientes do gradiente. Nos fragmentos florestais foram identificadas 130 espécies, seguido pelas áreas verdes com 103, escolas com 81 e centros de reciclagem com 46 , representando $92.8 \%, 73.6 \%, 57.8 \%$ e $32.8 \%$ da riqueza total, respectivamente. Os fragmentos florestais também apresentaram o maior número de ocorrências $(n=2851 ; 32.4 \%)$, seguido pelas áreas verdes $(n=2750 ; 31.3 \%)$, escolas $(n=2385$; $27.1 \%)$ e centros de reciclagem ( $n=804 ; 9.2 \%)$ (Informação Suplementar, Tabela S2). Essas diferenças ficam mais evidentes no padrão obtido a partir da riqueza das réplicas de cada tipo de ambiente conforme demonstrado na Figura 1. A análise de rarefação (Figura 2) demonstrou que as diferenças de riqueza entre os quatro ambientes foram significativas.

Apenas nove espécies se destacaram entre as mais abundantes nos ambientes amostrados. Duas delas pertencentes ao gênero Pheidole (Pheidole dyctiota Kempf, 1972, e Pheidole pubiventris Mayr, 1887) apareceram entre as cinco espécies mais abundantes nos quatro tipos de ambientes. Dorymyrmex brunneus Forel, 1908 e Nylanderia fulva, Mayr 1862, também se destacaram em

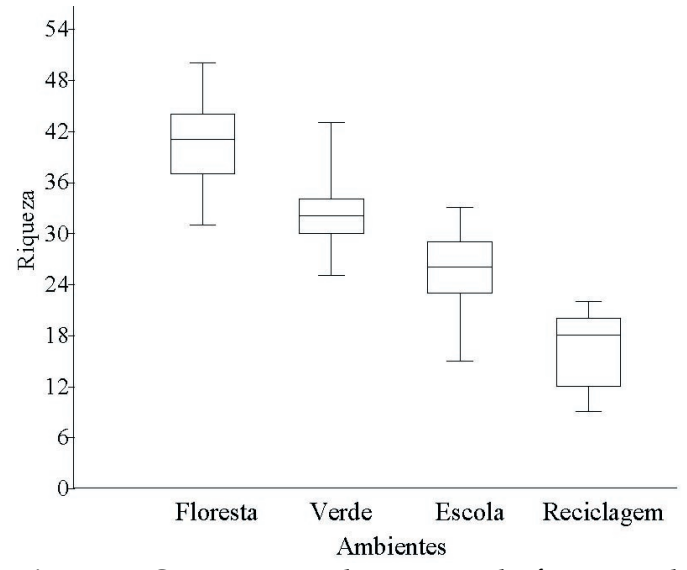

Figura 1. Comparativo da riqueza de formigas das réplicas de quatro ambientes (Floresta: fragmentos florestais; Verde: áreas verdes; Escola: ambientes escolares; Reciclagem: centros de reciclagem) de dez cidades situadas no Bioma Mata Atlântica, SC, sul do Brasil (fevereiro/março e novembro/dezembro de 2011). Os valores da riqueza estão apresentados na forma de "boxplots" que representam os valores das medianas, $1^{\circ}$ e $3^{\circ}$ quartis e valores extremos.

Figure 1. Richness comparison of ants from replicas of four environments (Floresta: forest fragments; Verde: green areas; Escola: schools environments; Reciclagem: recycling centers) at ten cities located in the Atlantic Forest Biome, SC, southern Brazil (February/March and November/December 2011). Richness values are presented in boxplots that represent the median, $1^{\text {st }}$ and $3^{\text {rd }}$ quartiles, and extreme values.

todos os ambientes amostrados, exceto nos fragmentos florestais, onde Camponotus rufipes (Fabricius 1775) e Pachycondyla striata F.

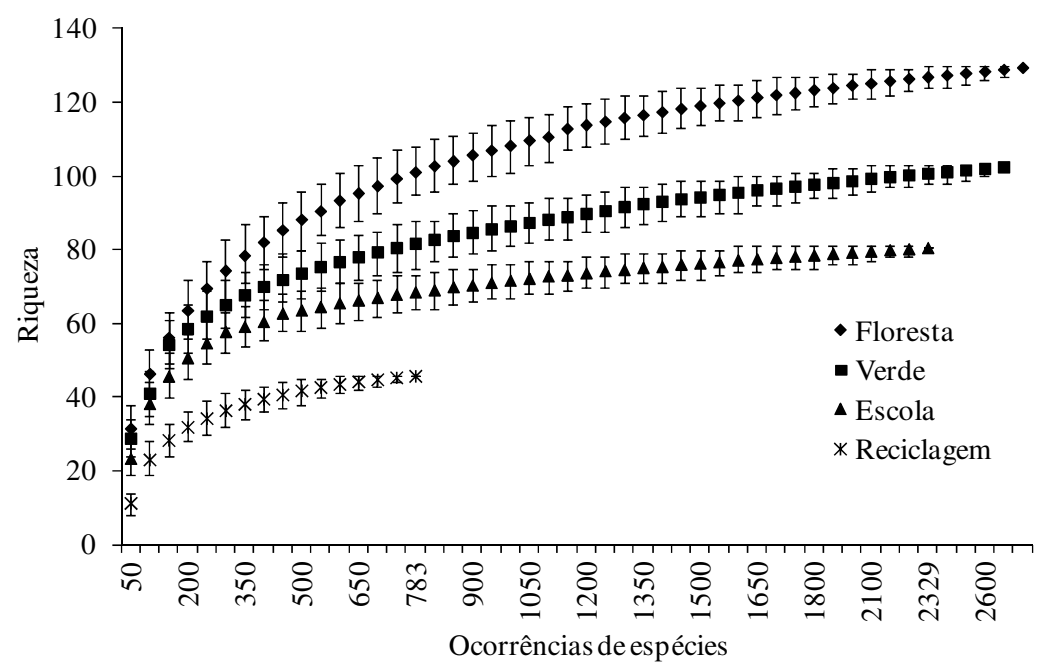

Figura 2. Comparação, pelo método de rarefação baseada no número de ocorrências, da fauna de formigas de quatro tipos de ambientes (Florestas: fragmentos florestais; Verdes: áreas verdes; Escolas: ambientes escolares; Reciclagem: centros de reciclagem) de dez cidades situadas no Bioma Mata Atlântica, SC, sul do Brasil (fevereiro/março e novembro/ dezembro de 2011). As barras indicam o intervalo de confiança (95\%).

Figure 2. Comparison by the rarefaction method based on occurrences number of ant fauna from four environments (Florestas: forest fragments; Verdes: green areas; Escolas: schools environments; Reciclagem: recycling centers) at ten cities in Atlantic Forest Biome, SC, southern Brazil (February/March and November/December 2011). The bars indicate the confidence interval $(95 \%)$. 
Tabela 1. Lista das espécies de formigas mais abundantes (a) e dominantes $\left(^{*}\right)$ com suas respectivas frequências relativas percentuais (\%) nas amostras em quatro tipos de ambiente (Floresta: fragmentos florestais; Verde: áreas verdes; Escolas: ambientes escolares; Reciclagem: centros de reciclagem) de dez cidades situadas no Bioma Mata Atlântica, SC, sul do Brasil (fevereiro/março e novembro/dezembro de 2011).

Table 1. List of the most abundant (a) and dominant $\left(^{*}\right)$ ant species with their respective relative percentage frequencies (\%) in the samples from four environments (Floresta: forest fragments; Verde: green areas; Escola: schools environments; Reciclagem: recycling centers) at ten cities in Atlantic Forest Biome, SC, southern Brazil (February/March and November/December 2011).

\begin{tabular}{lcccc}
\hline Táxon & Floresta & Verde & Escola & Reciclagem \\
\hline Camponotus mus Roger, 1863 & 1.9 & 3.3 & $4.3(\mathrm{a})$ & 3.2 \\
Camponotus rufipes (Fabricius, 1775) & $7.5(\mathrm{a})$ & 3.8 & 2.7 & 3.0 \\
Dorymyrmex brunneus Forel, 1908 & 0.9 & $4.0(\mathrm{a})$ & $5.7(\mathrm{a})$ & $11.3(\mathrm{a})\left(^{*}\right)$ \\
Nylanderia fulva (Mayr, 1862) & 2.7 & $5.4(\mathrm{a})$ & $11.0(\mathrm{a})\left({ }^{*}\right)$ & $9.8(\mathrm{a})$ \\
Pachycondyla striata F. Smith, 1858 & $4.0(\mathrm{a})$ & 3.2 & 0.7 & 0.0 \\
Pheidole dyctiota Kempf, 1972 & $5.9(\mathrm{a})$ & $11.7(\mathrm{a})\left(^{*}\right)$ & $11.7(\mathrm{a})\left(^{*}\right)$ & $15.5(\mathrm{a})\left(^{*}\right)$ \\
Pheidole pubiventris Mayr, 1887 & $8.0(\mathrm{a})$ & $10.1(\mathrm{a})\left(^{*}\right)$ & $9.9(\mathrm{a})$ & $8.1(\mathrm{a})$ \\
Pheidole tristis (F. Smith, 1858) & $9.8(\mathrm{a})$ & $4.3(\mathrm{a})$ & 3.0 & 2.2 \\
Solenopsis sp. 1 & 1.1 & 1.9 & 2.6 & $5.7(\mathrm{a})$ \\
\hline
\end{tabular}

Smith, 1858, apareceram entre as espécies mais abundantes. No ambiente escolar observou-se a espécie Camponotus mus Roger, 1863, entre as mais abundantes, enquanto nos centros de reciclagem se destacou a espécie Solenopsis sp. 1 (Tabela 1).

Nenhuma espécie foi classificada como dominante para os fragmentos florestais, segundo o critério adotado. Já nas áreas verdes foram classificadas como dominantes, as espécies $P$. pubiventris e $P$. dyctiota, sendo esta última dominante também nas escolas e nos centros de reciclagem. Ainda se destacaram como dominantes a espécie $N$. fulva no ambiente escolar e $D$. brunneus nos centros de reciclagem (Tabela 1 ).

Quanto às espécies mais raras, 18 tiveram apenas uma ocorrência e 13, duas ocorrências na soma da abundância dos quatro ambientes. Essas 31 espécies representam $22.1 \%$ da riqueza total inventariada. A assembleia de formigas dos fragmentos florestais contribuiu com o maior número de espécies (18) nesta condição, seguida pela assembleia das áreas verdes (12) e dos ambientes escolares (3) (Informação Suplementar, Tabela S2). Todas as espécies registradas nos centros de reciclagem apresentaram mais de duas ocorrências nas amostras.

Não houve substituição de espécies nos diferentes ambientes avaliadas. $\mathrm{O}$ teste NODF demonstrou que as assembleias de formigas amostradas nos fragmentos florestais, áreas verdes, escolas e centros de reciclagem são aninhadas (NODF observada: 95.1; NODF randomizada:68.8; SD: 7.0), ou seja, as três últimas assembleias podem ser consideradas subconjuntos da assembleia de formigas encontrada nos fragmentos florestais. A matriz de distribuição das espécies (Figura 3) demonstra o padrão de aninhamento encontrado.

Os fragmentos florestais apresentaram o maior número de espécies indicadoras (12), seguido pelas áreas verdes com quatro e escolas com três. Nos fragmentos florestais se destacaram Gnamptogenys striatula Mayr, 1884 , P. striata e Pheidole tristis (F. Smith 1858) com valor de indicação (VI) superior a 50\%, enquanto $P$. dyctiota apresentou o maior valor de indicação (32.8\%) nas áreas verdes e $N$. Fulva (35.7\%) nas escolas (Tabela 2). Nenhuma espécie apresentou valor de indicação maior ou igual a $25 \%$ nos centros de reciclagem.

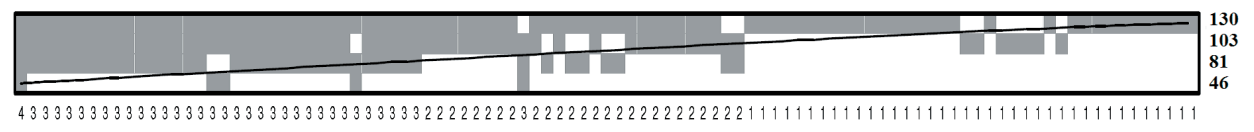

Figura 3. Matriz de distribuição de espécies de formigas no gradiente de quatro tipos de ambientes (fragmentos florestais, áreas verdes, escolas e centros de reciclagem) de dez cidades situadas no Bioma Mata Atlântica, SC, sul do Brasil (fevereiro/março e novembro/dezembro de 2011). Os ambientes estão dispostos nas linhas e as espécies nas colunas. A linha em diagonal indica a temperatura da matriz.

Figure 3. Ant species distribution matrix in a gradient of four environments (forest fragments, green areas, schools and recycling centers) at ten cities in Atlantic Forest Biome, SC, southern Brazil (February/March and November/ December 2011). The environments are arranged in rows and species in columns. The diagonal line indicates the temperature of the matrix. 
Tabela 2. Lista das espécies de formigas com Valor de Indicação (VI) superior a 25\% e probabilidade estatística $P<0.05$ associadas a três tipos de ambientes (Floresta: fragmentos florestais; Verde: áreas verdes; Escolas: ambientes escolares) de dez cidades situadas no Bioma Mata Atlântica, SC, sul do Brasil (fevereiro/março e novembro/dezembro de 2011). Table 2. List of ant species with indication value (VI) over $25 \%$ and statistical probability $P<0.05$ associated with three environments (Floresta: forest fragments; Verde: green areas; Escola: schools environments) at ten cities in Atlantic Forest Biome, SC, southern Brazil (February/March and November/December 2011).

\begin{tabular}{|c|c|c|c|c|}
\hline Táxon & VI(\%) Floresta & VI(\%) Verde & VI(\%) Escola & $P$ \\
\hline Gnamptogenys striatula (Mayr, 1884) & 61.7 & & & 0.0002 \\
\hline Pachycondyla striata (F. Smith, 1858) & 51.8 & & & 0.0002 \\
\hline Pheidole tristis (F. Smith, 1858) & 50.7 & & & 0.0002 \\
\hline Solenopsis stricta (Emery, 1896) & 34.6 & & & 0.0002 \\
\hline Pseudomyrmex termitarius (F. Smith, 1855) & 34.2 & & & 0.0002 \\
\hline Solenopsis sp. 4 & 32.5 & & & 0.0002 \\
\hline Pseudomyrmex sp. 1 & 31.8 & & & 0.0002 \\
\hline Solenopsis sp. 2 & 30.5 & & & 0.0002 \\
\hline Pheidole lignicola (Mayr, 1887) & 26.5 & & & 0.0016 \\
\hline Crematogaster magnifica (Santschi, 1925) & 25.5 & & & 0.0002 \\
\hline Pheidole punctatissima (Mayr, 1870) & 25.2 & & & 0.0034 \\
\hline Pheidole sp. 4 & 25.1 & & & 0.0006 \\
\hline Pheidole dyctiota (Kempf, 1972) & & 32.8 & & 0.0004 \\
\hline Pheidole pubiventris (Mayr, 1887) & & 29.6 & & 0.026 \\
\hline Camponotus crassus (Mayr, 1862) & & 28.6 & & 0.0004 \\
\hline Camponotus melanoticus (Emery, 1894) & & 28.6 & & 0.0002 \\
\hline Nylanderia fulva (Mayr, 1862) & & & 35.7 & 0.0002 \\
\hline Camponotus mus (Roger, 1863) & & & 31.3 & 0.001 \\
\hline Pheidole laevifrons (Mayr, 1887) & & & 25.2 & 0.0024 \\
\hline
\end{tabular}

\section{DISCUSSÃO}

A riqueza e a abundância de formigas variaram de um ambiente para outro apresentando tendência de redução de acordo com a intensificação do nível de perturbação antrópica. A abundância e a dominância caracterizaram de forma diferente as assembleias de formigas de cada ambiente. As espécies classificadas como abundantes e dominantes nos ambientes mais conservados não apresentaram tal condição nos ambientes mais perturbados. Não houve variação da composição das espécies entre uma assembleia e outra, mas houve perda de espécies ao longo do gradiente. O número de espécies com potencial de indicação decresceu à medida que aumenta o nível de perturbação antrópica do gradiente.

A maior riqueza e abundância observadas nos fragmentos florestais e nas áreas verdes estão de acordo com a hipótese da heterogeneidade ambiental (Pianka 1994), que prevề aumento da riqueza e da diversidade à medida que os ambientes tornam-se mais complexos e a oferta de recursos para o estabelecimento das espécies é maior. O potencial dos fragmentos florestais e das áreas verdes para a conservação de espécies de formigas em áreas urbanas já foi objeto de pesquisas anteriores (Morini et al. 2007; Pacheco \& Vasconcelos 2007) e os resultados deste estudo corroboram os resultados desses pesquisadores já que esses ambientes apresentaram maiores riquezas e abundâncias comparados aos ambientes escolares e aos centros de reciclagem. Os comparativos de riqueza ("boxplot" e análise de rarefação) mostraram diferenças entre as assembleias de formigas dos quatro ambientes do gradiente. Este resultado sugere que a diversidade de formigas de fragmentos florestais e de áreas verdes pode estar sendo favorecida por fatores como o tamanho das áreas, a cobertura vegetal, presença de serapilheira (existente nos fragmentos florestais) e pela menor intensidade das atividades humanas desenvolvida nesses ambientes. Apesar do menor esforço amostral nos centros de reciclagem, a redução de riqueza e de abundância de formigas nesse extremo do gradiente corrobora com Pacheco \& Vasconcelos (2007) e Sanford et al. (2008) cujos resultados demonstram que há uma redução significativa da diversidade de formigas em ambientes sob elevado nível de antropização quando comparados a ambientes sob menor perturbação.

Os fragmentos florestais apresentaram assembleias de formigas com a melhor distribuição da abundância entre as espécies, enquanto que nos centros de reciclagem foi constatada a maior concentração da abundância. Esse resultado corrobora com a predição de que a maior riqueza de um ambiente geralmente está associada com uma 
maior distribuição da abundância relativa das espécies (Ricklefs 2001). A maior abundância de C. mus, D. brunneus, N. fulva, P. dyctiota e $P$. pubiventris nos ambientes escolares e nos centros de reciclagem pode ser explicada pela tolerância que estas espécies possuem para se estabelecer sob tais condições ambientais. Espécies destes gêneros são geralmente classificadas como generalistas e oportunistas e toleram ambientes abertos, desprovidos de vegetação (Silvestre et al. 2003; Morini et al. 2007). Por outro lado, espécies mais raras nas amostras como Labidus praedator (F. Smith 1858), Camponotus hedwigae Forel, 1912, Pachycondyla marginata (Roger 1861), Pseudomyrmex gracilis (Fabricius, 1804) e Pseudomyrmex schuppi (Forel, 1901), tiveram ocorrências exclusivamente nas áreas verdes ou nos ambientes escolares e podem estar em situação de maior risco de desaparecerem localmente já que são formigas que dependem da vegetação ou de serrapilheira para a construção dos seus ninhos e para a obtenção de suas fontes de alimento (Silvestre et al. 2003).

Somente as áreas verdes, ambientes escolares e centros de reciclagem apresentaram espécies dominantes. Espécies generalistas como algumas pertencentes aos gêneros Pheidole, Nylanderia e Dorymyrmex (Silvestre et al. 2003) possuem características biológicas e ecológicas que favorecem a exploração de ambientes antropizados. Essas espécies não encontram limitações para a obtenção de fontes de nutrientes e de locais para nidificação nesses ambientes (Fowler et al. 1994). A condição de dominância observada nas áreas verdes e, especialmente nas escolas e nos centros de reciclagem, permite inferir que os ambientes sob maior perturbação antrópica favorecem a colonização por espécies tolerantes.

Apesar da ocorrência de algumas espécies exclusivamente nas áreas verdes ou nos ambientes escolares, as diferenças de riquezas entre as quatro assembleias não se relacionam com a substituição de espécies de um tipo de ambiente para outro (diversidade $\beta$ ) e sim com perdas de espécies ao longo do gradiente. Maior riqueza é encontrada em ambientes mais conservados, porém apenas espécies resistentes podem se manter em ambientes em condições mais restritivas (Townsend et al. 2003). Assim, é esperado que a fauna presente no extremo mais restritivo do gradiente seja constituída por uma fração daquelas espécies existentes no ambiente menos restritivo. Dentre os gêneros que se destacaram pela riqueza no estudo e que sofreram as maiores perdas de espécies ao longo do gradiente foram Pachycondyla (100\%), Pseudomyrmex (100\%), Crematogaster (87.5\%) e Camponotus (60\%). Azteca, Cephalotes, Cyphomyrmex, Hopoponera e Procryptocerus também tiveram perdas de $100 \%$ embora suas riquezas tenham sido menores (entre três e quatro espécies cada). As perdas de espécies em gêneros reconhecidos pela sua dependência de ambientes mais conservados corroboram Baselga (2010) que afirma que o padrão de aninhamento ocorre em função de uma perda não aleatória de espécies.

As espécies de formigas dos gêneros Pheidole e Solenopsis são descritas como generalistas e oportunistas que dominam a fonte de alimento (Silvestre \& Silva 2001). Seus valores de indicação podem estar associadas às técnicas de amostragem (iscas) aplicadas onde foram coletadas com muita frequência. A associação de espécies como G. striatula, P. striata, Pseudomyrmex termitarius (F. Smith 1855) e Crematogaster magnifica Santschi, 1925, com o ambiente dos fragmentos florestais pode ser explicado pela ecologia (alimentação e nidificação) dessas espécies que está associada à vegetação e ao solo em melhores condições de conservação (Silvestre et al. 2003).

Formigas do gênero Camponotus possuem tamanho médio a grande, são onívoras na maioria e nidificam em troncos podres, na vegetação ou mesmo no solo (Silvestre \& Silva 2001) e são frequentemente encontradas em áreas abertas (Iop et al. 2009). Estes hábitos podem ajudar a explicar a associação de Camponotus crassus Mayr, 1862, e Camponotus melanoticus Emery, 1894, como indicadoras nas áreas verdes e C. mus nas escolas. A associação de $N$. fulva como indicadora nas escolas pode ser explicada pela tolerância desta às perturbações antrópicas. Esta espécie é descrita como invasora, com distribuição cosmopolita.

A mais recente lista de formigas descritas para o estado de Santa Catarina (Ulysséa et al. 2011) relata a ocorrência de 207 espécies para a região onde o presente estudo foi realizado. Contudo, esta lista é o resultado de mais de seis décadas de pesquisas que utilizaram um conjunto amplo de técnicas de amostragem em diferentes substratos. O número de 130 espécies com ocorrência em pequenos fragmentos florestais inseridos em áreas urbanas proporciona uma ideia da fração da fauna de formigas que está encontrando nicho 
de sobrevivência neste tipo de ambiente. Nesse contexto, fragmentos de vegetação nativa e áreas verdes remanescentes de loteamentos tornam-se de fundamental interesse para a conservação das espécies (Silva et al. 2009), uma vez que esses ambientes podem ser reservatórios para o repovoamento de outros ambientes.

É preciso destacar a ocorrência das 81 espécies nos ambientes escolares e as 46 nos centros de reciclagem para a conservação da mirmecofauna em áreas urbanas. Apesar da perda de espécies em relação aos ambientes mais conservados, as escolas abrigam uma riqueza importante que incluiu a maioria dos gêneros e subfamílias amostrados no estudo. Todos os gêneros de formigas com ocorrência nos centros de reciclagem possuem espécies caracterizadas pela tolerância a ambientes abertos e sob perturbação antrópica (Silvestre et al. 2003). Dorymyrmex, Linepithema, Monomorium, Nylanderia, Paratrechina, Pheidole, Solenopsis e Wasmannia possuem espécies descritas como invasoras, tolerantes às perturbações ambientais e frequentemente encontradas em ambientes urbanos. Camponotus, Acromyrmex e Crematogaster se associam com a vegetação e a ocorrência de espécies destes gêneros nos centros de reciclagem pode indicar que estas espécies estão incluindo estes ambientes na sua área de forrageio.

Neste estudo, foram encontradas variações significativas de riqueza e de abundância de formigas no gradiente de perturbação antrópica avaliado mostrando uma tendência de redução desses parâmetros nos ambientes escolares e centros de reciclagem (mais antropizados) em relação aos fragmentos florestais e áreas verdes (menos antropizados). Os resultados demonstraram também uma tendência de aumento na dominância de espécies à medida que as ações antrópicas se intensificam, além de uma substituição das espécies mais abundantes ao longo do gradiente. Observou-se que as assembleias de formigas não se alteram em sua composição, mas que há uma perda importante de espécies de um ambiente para outro. Considerando a importância ecológica das formigas e a existência de uma diversidade de plantas e de outros animais que a presença das formigas permite supor, os resultados podem contribuir para a implementação de programas de educação ambiental e de conservação de fragmentos florestais e de áreas verdes em áreas urbanas.
Agradecimentos: À Coordenação de Aperfeiçoamento de Pessoal de Nível Superior (CAPES) pela concessão da bolsa de estudos para o desenvolvimento desta pesquisa. Ao Sistema de Autorização e Informação em Biodiversidade (SISBIO) pela autorização número 24174-1 para a realização das amostragens. Aos proprietários das áreas particulares e aos gestores das áreas verdes, escolas e dos centros de reciclagem pela permissão das coletas. À bióloga G Najar Porto pelo auxílio nas atividades de coleta, acondicionamento e triagem das formigas amostradas.

\section{REFERENNCIAS}

Almeida-Neto, M; P Guimarães; PR Guimarães; RD Loyola \& W UlRICH. 2008. A consistent metric for nestedness analysis in ecological systems: reconciling concept and measurement. Oikos, 117:1227-1239.

Alonso, LE \& D Agosti. 2000. Biodiversity studies, monitoring, and ants: an overview. Pp. 1-8 em: Agosti, D ;JD MajER; LE Alonso \& TR Schultz (eds.). Ants: standard methods for measuring and monitoring biodiversity. Capítulo 1. Smithsonian Institution. Washington. Pp. 280.

AtMAR, W \& BD PATterson. 1993. The measure of order and disorder in the distribution of species in fragmented habitat. Oecologia, 96:373-382.

BASELGA, A. 2010. Partitioning the turnover and nestedness components of beta diversity. Global Ecology and Biogeography, 19:134-143.

Bestelmeyer, BT; D Agosti; LE Alonso; CRF Brandẽo; WL BROWN JR; ET AL. 2000. Field techniques for the study of ground-dwelling ants. Pp. 122-144 em: Agosti, D; JD Majer; LE Alonso \& TR Schultz (eds.). Ants: standard methods for measuring and monitoring biodiversity. Capítulo 9. Smithsonian Institution. Washington. Pp. 280.

Buczckowski, G \& DS Richmond. 2012. The effect of urbanization on ant abundance and diversity: a temporal examination of factors affecting biodiversity. PLOS ONE, 7:e41729.

CONNOR, EF; J HAFERniK; VL MOORE \& JK RICKMAN. 2002. Insect conservation in an urban biodiversity hotspot: the San Francisco Bay area. Journal of Insect Conservation, 6:247-259.

DÁtTilo, W; N Sibinel; JCF FAlCão \& RV Nunes. 2011. Mirmecofauna em um fragmento de floresta atlântica urbana no município de Marília, SP, Brasil. Bioscience Journal, 27:494-504.

DiAmond, J. 2012. Colapso. $8^{\text {a }}$. Edição. Editora Record. Rio de Janeiro. Pp. 699.

DufrêNE, M \& P LEGENDRE. 1997. Species assemblages and indicator species: The need for a flexible asymetrical aproach. Ecological Monographs, 67:345-366.

Fowler, HG; MN SCHLINDWEIN \& MA Medeiros. 1994. Exotic ants and community simplification in Brazil: a review of the impact of exotic ants on native ant assemblages. Pp. 151-162 em: Williams, DF (ed.). Exotic ants: Biology, Impact, and Control of Introduced species. Boulder Westview Press. Pp. 352.

Gotelli, NJ \& GL EnTSMinger. 2001. EcoSim: Null models software for ecology. Versão 7.0. Acquired Intelligence Inc. \& Kesey-Bear. http://homepages.together.net/ gentsmin/ecosim.htm (último acesso: 17/03/2013).

GotelLI, NJ \& RK COLWELL. 2001. Quantifying biodiversity: procedures and pitfalls in the measurement and comparison of species richness. Ecology Letters, 4:379-391. 
Hammer, O; DAT Harper \& PD Rian. 2001. Past: Palaeonthological statistics software package for education and data analysis. Versão. 1.37. http: //palaeo-electronica.org/2001_1/past/issue1_01.htm (último acesso: 21/11/2013).

Hölldobler, B \& EO Wilson. 1990. The ants. Cambridge: Belknap, Harvard University Press. Pp. 732.

IBGE (Instituto Brasileiro de Geografia e Estatística). 2010. http:/ / www.ibge.gov.br/cidadesat/default.php (último acesso: 19/11/2013).

Iop, S; VM Caldart; JA Lutinski \& FRM Garcia. 2009. Formigas urbanas da cidade de Xanxerê, Santa Catarina, Brasil. Biotemas, 22:55-64.

López-Moreno, IR; ME Diaz-Betancourt \& TS Landa. 2003. Insectos sociales em ambientes antropizados: las hormigas de la ciudad de Coatepec, Veracruz, México. Sociobiology, 42:605-622.

LuTinSKI, JA; CJ LuTinSKI; S Iop \& FRM Garcia. 2013a. Evaluation of an ant sampling protocol (Hymenoptera: Formicidae) in three modified environments located inside an austral Atlantic Forest area of Brazil. Ecología Austral, 23:37-43.

LutinSKI, JA; BC LoPes \& ABB Morais. 2013b. Diversidade de formigas urbanas (Hymenoptera: Formicidae) de dez cidades do sul do Brasil. Biota Neotropica, 13:1-12.

LuTinsKI, JA \& MF SouZA. 2009. Avaliação do sistema de coleta de resíduos recicláveis realizado por catadores e suas implicações sociais, econômicas, ambientais e sanitárias na cidade de Chapecó - SC. Monografia de Pós Graduação em Biotecnologia e Energias Alternativas, FAISA. Pp. 56.

McCune, B \& MJ MefFord. 2006. PC-ORD. Multivariate analysis of ecological data. MjM Software, Gleneden Beach, Ore.

MCKINNEY, ML. 2002. Urbanization, biodiversity, and conservation. BioScience, 52(10):883-890.

Melo, AS; RAS Pereira; AJ Santos; GJ Shepherd; G MACHADO; ET AL. 2003. Comparing species richness among assemblages using sample units: why not use extrapolation methods to standardize different sample sizes? Oikos, 101:398-410.

Morini, MSC; CB Munhae; R Leung; DF Candiani \& JC Voltolini. 2007. Comunidades de formigas (Hymenoptera, Formicidae) em fragmentos de Mata Atlântica situados em áreas urbanizadas. Iheringia, Série Zoologia, 97:246-252.

NOVOTNÝ, V \& Y BASSET. 2000. Rare species in communities of tropical insect herbivores: pondering the mistery of singletons. Oikos, 89:564-572.

Oliveira, MF \& AEC CAmpos-Farinha. 2005. Formigas urbanas do município de Maringá, PR, e suas implicações. Arquivos do Instituto Biológico, 72:33-39.

Pacheco, R \& HL VAsconcelos. 2007. Invertebrate conservation in urban areas: ants in the Brazilian Cerrado. Landscape and Urban Planning, 81:193-199.
PianKa, E. 1994. Evolutionary ecology. New York: Harper Collins College Publishers. Pp. 484.

R Development Core Team. 2011. R: A language and environment for statistical computing. R Foundation for Statistical Computing, Vienna, Austria. Disponível em http://www.R-project.org (último acesso: 20/10/2011).

RickLefs, RE. 2001. A Economia da Natureza. Editora Guanabara. Pp. 572.

SANFORD, MP; PN MANLEY \& DD MuRPHy. 2008. Effects of urban development on ant communities: implications for ecosystem services and management. Conservation Biology, 23:131-141.

SARMIENTO, CE. 2003. Metodologías de captura y estudio de las hormigas. Hormigas como herramienta para la bioindicación y monitoreo. Pp. 201-210 em: FERNÁNDEZ, $\mathrm{F}$ (ed.). Introducción a las hormigas de la región neotropical. Capítulo 12. Instituto de Investigación de Recursos Biológicos Alexander von Humboldt. Bogotá, Colombia. Pp. 424.

Silva, RS; DMN Passos \& AC ReZende. 2009. Biogeografia urbana: estudo de comunidades de formigas nos parques municipais de Belo Horizonte - MG. Anais do III Congresso Latino Americano de Ecologia. 10 a 13 de Setembro de 2009, São Lourenço - MG.

Silvestre R; CRF Brandão \& RR Silva. 2003. Grupos funcionales de hormigas: el caso de los gremios del cerrado. Pp. 159-166 em: FeRNÁNDEZ, F (ed.). Introducción a las hormigas de la región neotropical. Capítulo 7. Instituto de Investigación de Recursos Biológicos Alexander von Humboldt. Bogotá, Colombia. Pp. 424.

Silvestre, R \& RR Silva. 2001. Guildas de formigas da estação ecológica do Jataí, Luiz Antônio -SP- sugestões para a aplicação do modelo de guildas como bioindicadores ambientais. Biotemas, 14:37-69.

Stringer, LD; AEA STEPHENS; DM SUCKLING \& JG ChARLES. 2009. Ant dominance in urban areas. Urban Ecosystems, 12:503-514.

Toennisson, TA; NJ SAnders; WE Klingeman \& KM VAIL. 2011. Influences on the structure of suburban ant (Hymenoptera: Formicidae) communities and the abundance of Tapinoma sessile. Environmental Entomology 40:1397-1404.

Townsend, CR; M Begon \& JL HaRPer. 2003. Fundamentos em ecologia. Artmed, Porto Alegre. Pp. 592.

Tukey, JW. 1977. Exploratory Data Analysis. Addison Wesley Longman Inc. Pp. 688.

Ulrich, W; M Almeida-Neto \& NJ Gotelli. 2009. A consumer's guide to nestedness analysis. Oikos, 118: 3-17.

Ulysséa, MA; CE CERETO; FB RosumeK; RR Silva \& BC LOPES. 2011. Updated list of ant species (Hymenoptera, Formicidae) recorded in Santa Catarina State, southern Brazil, with a discussion of research advances and priorities. Revista Brasileira de Entomologia, 55:603-611. 


\section{INFORMAÇÕES SUPLEMENTARES}

Tabela S1. Coordenadas geográficas, população urbana (Pop. urb.) e número de réplicas de quatro ambientes (Floresta: fragmentos florestais; Verde: áreas verdes; Escolas: ambientes escolares; Reciclagem: centros de reciclagem) de dez cidades situadas no Bioma Mata Atlântica, SC, Sul do Brasil, onde o estudo foi conduzido (fevereiro/março e novembro/dezembro de 2011).

Table S1. Geographic coordinates, urban population (Pop urb.) and number of replicas of four environments (Floresta: forest fragments; Verde: green areas; Escola: schools environments; Reciclagem: recycling centers) of ten cities located in the Atlantic Forest Biome, SC, southern Brazil, where the study was conducted (February/March and November/ December 2011).

\begin{tabular}{|c|c|c|c|c|c|c|}
\hline Cidades & Coordenadas & Pop. urb. & Floresta & Verde & Escola & Reciclagem \\
\hline Abelardo Luz & $26^{\circ} 33^{\prime} 58^{\prime \prime}$ S e $52^{\circ} 19^{\prime} 40^{\prime \prime} \mathrm{W}$ & 9570 & 3 & 3 & 3 & 1 \\
\hline Campo Erê & $26^{\circ} 23^{\prime} 39^{\prime \prime}$ S e $53^{\circ} 04^{\prime} 44^{\prime \prime} \mathrm{W}$ & 6252 & 3 & 3 & 3 & 2 \\
\hline Chapecó & $27^{\circ} 05^{\prime} 51^{\prime \prime}$ S e $52^{\circ} 38^{\prime} 31^{\prime \prime} \mathrm{W}$ & 168113 & 3 & 3 & 3 & 3 \\
\hline Concórdia & $27^{\circ} 13^{\prime} 32^{\prime \prime}$ S e $52^{\circ} 01^{\prime} 14^{\prime \prime} \mathrm{W}$ & 54865 & 3 & 3 & 3 & 2 \\
\hline Joaçaba & $27^{\circ} 10^{\prime} 01^{\prime \prime}$ S e $51^{\circ} 29^{\prime} 48^{\prime \prime} \mathrm{W}$ & 24924 & 3 & 3 & 3 & 2 \\
\hline Palmitos & $27^{\circ} 04^{\prime} 06^{\prime \prime}$ S e $53^{\circ} 09^{\prime} 46^{\prime \prime} \mathrm{W}$ & 9871 & 3 & 3 & 3 & 2 \\
\hline Pinhalzinho & $26^{\circ} 50^{\prime} 53^{\prime \prime}$ S e $52^{\circ} 59^{\prime} 20^{\prime \prime} \mathrm{W}$ & 13615 & 3 & 3 & 3 & 2 \\
\hline São Miguel do Oeste & $26^{\circ} 43^{\prime} 32^{\prime \prime}$ S e $53^{\circ} 30^{\prime} 59^{\prime \prime} \mathrm{W}$ & 32065 & 3 & 3 & 3 & 2 \\
\hline Seara & $27^{\circ} 08^{\prime} 57^{\prime \prime}$ S e $52^{\circ} 18^{\prime} 39^{\prime \prime} \mathrm{W}$ & 11586 & 3 & 3 & 3 & 0 \\
\hline Xanxerê & $26^{\circ} 52^{\prime} 38^{\prime \prime}$ S e $52^{\circ} 24^{\prime} 18^{\prime \prime} \mathrm{W}$ & 39143 & 3 & 3 & 3 & 3 \\
\hline Total & _ & - & 30 & 30 & 30 & 19 \\
\hline
\end{tabular}

* IBGE (2010).

Tabela S2. Lista das espécies de formigas com suas respectivas ocorrências em quatro ambientes: (Floresta: fragmentos florestais; Verde: áreas verdes; Escola: ambientes escolares; Reciclagem: centros de reciclagem) de dez cidades situadas no Bioma Mata Atlântica, SC, sul do Brasil (fevereiro/março e novembro/dezembro de 2011).

Table S2. List of ant species with their respective occurrences in four environments (Floresta: forest fragments; Verde: green areas; Escola: schools environments; Reciclagem: recycling centers) at ten cities in Atlantic Forest Biome, SC, southern Brazil (February/March and November/December 2011).

\begin{tabular}{|c|c|c|c|c|}
\hline Táxon & Floresta & Verde & Escola & Reciclagem \\
\hline \multicolumn{5}{|l|}{ Subfamília Cerapachyinae } \\
\hline \multicolumn{5}{|l|}{ Tribo Acanthostichini } \\
\hline Acanthostichus quadratus Emer, 1895 & 1 & & & \\
\hline \multicolumn{5}{|l|}{ Subfamília Dolichoderinae } \\
\hline \multicolumn{5}{|l|}{ Tribo Dolichoderini } \\
\hline Azteca instabilis (F. Smith, 1862) & 1 & & & \\
\hline Azteca muelleri Emery, 1893 & 1 & & & \\
\hline Dorymyrmex brunneus Forel, 1908 & 26 & 109 & 135 & 91 \\
\hline Dorymyrmex pyramicus (Roger, 1863) & 6 & 24 & 42 & 20 \\
\hline Dorymyrmex sp. & 1 & & & \\
\hline Linepithema angulatum (Emery, 1894) & 1 & & & \\
\hline Linepithema gallardoi Kusnezov, 1969 & 35 & 15 & 7 & 3 \\
\hline Linepithema humile (Mayr, 1868) & 73 & 50 & 89 & 14 \\
\hline Linepithema iniquum (Mayr, 1870) & 15 & 23 & 16 & 5 \\
\hline Linepithema leucomelas (Emery, 1894) & 6 & 5 & & 1 \\
\hline Linepithema micans (Forel, 1908) & 13 & 2 & 6 & 1 \\
\hline Tapinoma melanocephalum (Fabricius, 1793) & 28 & 6 & 12 & 2 \\
\hline \multicolumn{5}{|l|}{ Subfamília Ecitoninae } \\
\hline \multicolumn{5}{|l|}{ Tribo Ecitonini } \\
\hline Eciton quadriglume (Haliday, 1836) & 1 & & & \\
\hline Labidus praedator (F. Smith, 1858) & & 1 & & \\
\hline \multicolumn{5}{|l|}{ Subfamília Ectatomminae } \\
\hline \multicolumn{5}{|l|}{ Tribo Ectatommini } \\
\hline Ectatomma edentatum Roger, 1863 & 1 & & 2 & \\
\hline Gnamptogenys striatula Mayr, 1884 & 57 & & & \\
\hline Gnamptogenys sulcata (Smith, 1858) & 1 & 2 & & \\
\hline \multicolumn{5}{|l|}{ Subfamília Formicinae } \\
\hline \multicolumn{5}{|l|}{ Tribo Camponotini } \\
\hline Camponotus (Myrmaphaenus) blandus (F. Smith, 1858) & 1 & 1 & & \\
\hline
\end{tabular}


Tabela S2. Continuação

Table S2. Continuation.

\begin{tabular}{|c|c|c|c|c|}
\hline Táxon & Floresta & Verde & Escola & Reciclagem \\
\hline Camponotus (Myrmaphaenus) cameranoi Emery, 1894 & 8 & 9 & 9 & \\
\hline Camponotus (M.) fastigatus Roger, 1863 & 35 & 50 & 47 & 4 \\
\hline Camponotus (M.) novogranadensis Mayr, 1870 & 3 & 1 & & \\
\hline Camponotus (M.) personatus Emery, 1894 & 4 & 15 & 5 & 2 \\
\hline Camponotus (Myrmepomis) sericeiventris (G.-Méneville, 1838) & 2 & 5 & 2 & \\
\hline Camponotus (Myrmobrachys) crassus Mayr, 1862 & 22 & 61 & 37 & 5 \\
\hline Camponotus (M.) mus Roger, 1863 & 55 & 92 & 103 & 26 \\
\hline Camponotus (Myrmocladoecus) hedwigae Forel, 1912 & & 1 & & \\
\hline Camponotus (Myrmosphincta) sexguttatus (Fabricius, 1793) & 1 & & 2 & \\
\hline Camponotus (Myrmothrix) atriceps (F. Smith, 1858) & 4 & & & \\
\hline Camponotus (M.) cingulatus Mayr, 1862 & 2 & 4 & 1 & \\
\hline Camponotus (M.) rufipes (Fabricius, 1775) & 215 & 104 & 64 & 24 \\
\hline Camponotus (Pseudocolobopsis) alboannulatus Mayr, 1887 & 1 & 1 & & \\
\hline Camponotus (Tanaemyrmex) lespesii Forel, 1886 & 4 & 10 & 4 & \\
\hline Camponotus (Tanaemyrmex) melanoticus Emery, 1894 & 41 & 89 & 52 & 3 \\
\hline Camponotus (T.) punctulatus Mayr, 1868 & 33 & 39 & 56 & 9 \\
\hline Camponotus sp. 1 & 2 & 10 & 6 & \\
\hline Camponotus sp. 2 & 1 & 1 & & \\
\hline Camponotus sp. 3 & 13 & 10 & 6 & 2 \\
\hline Camponotus sp. 4 & 2 & 3 & 1 & \\
\hline \multicolumn{5}{|l|}{ Tribo Myrmelachistini } \\
\hline Myrmelachista catharinae Mayr, 1887 & 6 & 4 & & \\
\hline Myrmelachista catharinae maior Santschi, 1936 & 2 & & & \\
\hline Myrmelachista gagatina Emery, 1894 & 3 & 4 & 1 & \\
\hline Myrmelachista reticulata Borgmeier, 1928 & 2 & & & \\
\hline \multicolumn{5}{|l|}{ Tribo Plagiolepidini } \\
\hline Brachymyrmex (Brachymyrmex) aphidicola (Forel, 1909) & 11 & 12 & 2 & \\
\hline Brachymyrmex (B.) coactus Mayr, 1887 & 36 & 57 & 58 & 41 \\
\hline Brachymyrmex (B. cordemoyi Forel, 1895 & 14 & 2 & 1 & 1 \\
\hline Brachymyrmex (Bryscha) pilipes Mayr, 1887 & 4 & 1 & & \\
\hline Brachymyrmex sp. & 2 & 1 & & \\
\hline Nylanderia fulva (Mayr, 1862) & 78 & 149 & 263 & 79 \\
\hline Nylanderia sp. & 7 & 13 & 8 & 6 \\
\hline Paratrechina longicornis (Latreille, 1802) & 8 & 14 & & 2 \\
\hline \multicolumn{5}{|l|}{ Subfamília Heteroponerinae } \\
\hline \multicolumn{5}{|l|}{ Tribo Heteroponerini } \\
\hline Heteroponera flava Kempf, 1962 & 6 & 1 & & \\
\hline Heteroponera inermis (Emery, 1894) & 5 & & & \\
\hline \multicolumn{5}{|l|}{ Subfamília Myrmicinae } \\
\hline \multicolumn{5}{|l|}{ Tribo Attini } \\
\hline Acromyrmex (Acromyrmex) ambiguus (Emery, 1888) & 20 & 24 & 18 & 2 \\
\hline Acromyrmex (A.) aspersus (F. Smith, 1858) & 9 & 8 & 7 & \\
\hline Acromyrmex (A.) disciger (Mayr, 1887$)$ & 9 & 6 & 6 & \\
\hline Acromyrmex (A.) lundii (Guérin-Méneville, 1838) & 19 & 19 & 17 & 2 \\
\hline Acromyrmex (A.) niger (F. Smith, 1858) & 6 & 9 & 7 & 1 \\
\hline Acromyrmex (A.) rugosus (F. Smith, 1858) & 1 & & & \\
\hline Acromyrmex (A.) subterraneus (Forel, 1893) & 17 & 24 & 13 & \\
\hline Apterostigma mayri Forel, 1893 & 1 & & & \\
\hline Apterostigma pilosum Mayr, 1865 & 2 & 1 & 1 & \\
\hline Apterostigma wasmannii Forel, 1892 & 11 & & & \\
\hline Atta (Neoatta) sexdens (Linnaeus, 1758) & 9 & 2 & 5 & \\
\hline Atta sp. & 4 & 2 & 1 & \\
\hline Cyphomyrmex plaumanni Kempf, 1962 & 4 & & & \\
\hline Cyphomyrmex rimosus (Spinola, 1853) & 4 & 23 & 6 & \\
\hline Cyphomyrmex strigatus Mayr, 1887 & 2 & 2 & & \\
\hline Mycocepurus goeldii (Forel, 1893) & 8 & 7 & 3 & \\
\hline
\end{tabular}


Tabela S2. Continuação.

Table S2. Continuation.

\begin{tabular}{|c|c|c|c|c|}
\hline Táxon & Floresta & Verde & Escola & Reciclagem \\
\hline \multicolumn{5}{|l|}{ Tribo Blepharidattini } \\
\hline Wasmannia affinis Santschi, 1929 & 1 & & 1 & \\
\hline Wasmannia auropunctata (Roger, 1863) & 28 & 55 & 22 & 10 \\
\hline \multicolumn{5}{|l|}{ Tribo Cephalotini } \\
\hline Cephalotes angustus (Mayr, 1862) & 1 & & & \\
\hline Cephalotes pinelii (Guérin-Méneville, 1844) & 3 & & & \\
\hline Cephalotes pusillus (Klug, 1824) & 9 & 16 & 2 & \\
\hline Procryptocerus adlerzi (Mayr, 1887) & 6 & & & \\
\hline Procryptocerus convergens (Mayr, 1887) & 6 & & & \\
\hline Procryptocerus goeldii Forel, 1899 & 3 & & & \\
\hline \multicolumn{5}{|l|}{ Tribo Crematogastrini } \\
\hline Crematogaster (Eucrema) acuta (Fabricius, 1804) & 12 & 15 & 3 & 3 \\
\hline Crematogaster (E.) bingo Forel, 1908 & 27 & 7 & 4 & \\
\hline Crematogaster (Neocrema) corticicola Mayr, 1887 & 2 & 3 & & \\
\hline Crematogaster (N.) magnifica Santschi, 1925 & 24 & 8 & 1 & \\
\hline Crematogaster (Orthocrema) curvispinosa Mayr, 1862 & 3 & 3 & & \\
\hline Crematogaster sp. 1 & 21 & 75 & 64 & 18 \\
\hline Crematogaster sp. 2 & 21 & 19 & 4 & \\
\hline Crematogaster sp. 3 & 19 & 17 & 5 & \\
\hline \multicolumn{5}{|l|}{ Tribo Dacetini } \\
\hline Basiceros (Eurhopalothrix) convexiceps (Mayr, 1887) & 1 & & & \\
\hline Strumigenys cultrigera Mayr, 1887 & 1 & 1 & & \\
\hline \multicolumn{5}{|l|}{ Tribo Formicoxenini } \\
\hline Cardiocondyla nuda (Mayr, 1866) & 3 & 3 & 2 & \\
\hline Nesomyrmex sp. & & 2 & & \\
\hline Tribo Myrmicini & 5 & & & \\
\hline Pogonomyrmex angustus Mayr, 1870 & 11 & 16 & 21 & \\
\hline Pogonomyrmex naegelii Forel, 1878 & 2 & & & \\
\hline Pogonomyrmex sp. 1 & 7 & & & \\
\hline \multicolumn{5}{|l|}{ Pogonomyrmex sp. 2} \\
\hline \multicolumn{5}{|l|}{ Tribo Pheidolini } \\
\hline Pheidole (Elasmopheidole) aberrans Mayr, 1868 & 1 & & 1 & \\
\hline Pheidole (Pheidole) brevicona Mayr, 1887 & 8 & 6 & 8 & 2 \\
\hline Pheidole (P.) dyctiota Kempf, 1972 & 168 & 322 & 278 & 125 \\
\hline Pheidole (P.) laevifrons Mayr, 1887 & 20 & 49 & 81 & 10 \\
\hline Pheidole (P.) lignicola Mayr, 1887 & 85 & 64 & 32 & 14 \\
\hline Pheidole (P.) megacephala (Fabricius, 1793) & & 1 & 1 & \\
\hline Pheidole (P.) pubiventris Mayr, 1887 & 228 & 277 & 235 & 65 \\
\hline Pheidole (P.) punctatissima Mayr, 1870 & 72 & 47 & 35 & 14 \\
\hline Pheidole $(P$.$) risii Forel, 1892$ & 5 & 1 & 2 & \\
\hline Pheidole (P.) tristis (F. Smith, 1858) & 280 & 117 & 72 & 18 \\
\hline Pheidole sp. 1 & 44 & 23 & 25 & 10 \\
\hline Pheidole sp. 2 & 11 & & & \\
\hline Pheidole sp. 3 & 3 & 1 & & 21 \\
\hline Pheidole sp. 4 & 38 & 15 & 7 & 2 \\
\hline Pheidole sp. 5 & 39 & 34 & 27 & 4 \\
\hline \multicolumn{5}{|l|}{ Tribo Solenopsidini } \\
\hline Monomorium floricola (Jerdon, 1851) & & 1 & 1 & 1 \\
\hline Monomorium pharaonis (Linnaeus, 1758) & & 2 & & \\
\hline Solenopsis invicta Buren, 1972 & 29 & 80 & 94 & 30 \\
\hline Solenopsis saevissima (F. Smith, 1855) & 12 & 55 & 59 & 27 \\
\hline Solenopsis schmalzi Forel, 1901 & 2 & & 1 & \\
\hline Solenopsis stricta Emery, 1896 & 72 & 23 & 20 & 4 \\
\hline Solenopsis sp. 1 & 30 & 51 & 63 & 46 \\
\hline Solenopsis sp. 2 & 47 & 6 & 9 & 3 \\
\hline Solenopsis sp. 3 & 1 & & 2 & 1 \\
\hline
\end{tabular}


Tabela S2. Continuação.

Table S2. Continuation.

\begin{tabular}{|c|c|c|c|c|}
\hline Táxon & Floresta & Verde & Escola & Reciclagem \\
\hline Solenopsis sp. 4 & 101 & 26 & 33 & 30 \\
\hline Solenopsis sp. 5 & & 3 & 3 & \\
\hline Solenopsis sp. 6 & 2 & 3 & & \\
\hline \multicolumn{5}{|l|}{ Subfamília Ponerinae } \\
\hline \multicolumn{5}{|l|}{ Tribo Ponerini } \\
\hline Dinoponera australis Emery, 1901 & 3 & 2 & & \\
\hline Hypoponera distinguenda (Emery, 1890) & 2 & & & \\
\hline Hypoponera opaciceps (Mayr, 1887) & 1 & & & \\
\hline Hypoponera trigona (Mayr, 1887) & 5 & & & \\
\hline Hypoponera sp. & 1 & & & \\
\hline Odontomachus chelifer (Latreille, 1802) & 25 & 9 & 1 & \\
\hline Pachycondyla crenata (Roger, 1858) & 8 & & & \\
\hline Pachycondyla harpax (Fabricius, 1804) & 12 & 1 & & \\
\hline Pachycondyla marginata (Roger, 1861) & & 1 & & \\
\hline Pachycondyla striata F. Smith, 1858 & 114 & 89 & 17 & \\
\hline Pachycondyla villosa (Fabricius, 1804) & 5 & 1 & & \\
\hline Pachycondyla sp. & 4 & 1 & & \\
\hline \multicolumn{5}{|l|}{ Subfamília Pseudomyrmecinae } \\
\hline \multicolumn{5}{|l|}{ Tribo Pseudomyrmecini } \\
\hline Pseudomyrmex flavidulus (F. Smith, 1858) & 7 & 12 & 1 & \\
\hline Pseudomyrmex gracilis (Fabricius, 1804) & & 1 & & \\
\hline Pseudomyrmex phyllophilus (F. Smith, 1858) & 1 & & & \\
\hline Pseudomyrmex schuppi (Forel, 1901) & & 1 & & \\
\hline Pseudomyrmex termitarius (F. Smith, 1855) & 52 & 19 & 10 & \\
\hline Pseudomyrmex sp. 1 & 48 & 22 & 13 & \\
\hline Pseudomyrmex sp. 2 & 21 & 12 & 3 & \\
\hline Pseudomyrmex sp. 3 & 2 & 4 & 1 & \\
\hline Total de ocorrências & 2848 & 2750 & 2385 & 804 \\
\hline
\end{tabular}

